\title{
Pharmacological Management of Dementia with Lewy Bodies
}

\author{
Linda A. Hershey ${ }^{1}\left[\right.$ [ Rhonda Coleman-Jackson ${ }^{1}$
}

Published online: 25 January 2019

(c) The Author(s) 2019

\begin{abstract}
Dementia with Lewy bodies (DLB) is a complex disease that involves a variety of cognitive, behavioral and neurological symptoms, including progressive memory loss, visual hallucinations, parkinsonism, cognitive fluctuations and rapid eye movement sleep behavior disorder (RBD). These symptoms may appear in varying combinations and levels of severity in each patient who is seen in the clinic, making diagnosis and treatment a challenge. DLB is the third most common of all the neurodegenerative diseases behind both Alzheimer's disease and Parkinson's disease (PD). The median age of onset for DLB (76.3 years) is younger than that seen in PD dementia (81.4 years). New pathological studies have shown that most DLB patients have variable amounts of Alzheimer's changes in their brains, explaining the wide variability in this disease's clinical presentation and clinical course. This review discusses the three cholinesterase inhibitors that have been shown to be effective in managing the cognitive and behavioral symptoms of DLB: rivastigmine, galantamine and donepezil. Memantine is able to improve clinical global impression of change in those with mild to moderate DLB. Levodopa can treat the parkinsonism of some DLB patients, but the dose is often limited due to the fact that it can cause agitation or worsening of visual hallucinations. A recent phase 2 clinical trial showed the benefit of zonisamide when it is added as an adjunct to levodopa for treating DLB parkinsonism. While atypical antipsychotic drugs may not always be helpful as monotherapy in managing the agitation associated with DLB, low doses of valproic acid can be effective when added as an adjunct to drugs like quetiapine. Pimavanserin may prove to be a useful treatment for psychosis in DLB patients, but like other antipsychotic drugs that are used in dementia patients, there is a small increased risk of mortality. RBD, which is a common core clinical feature of DLB, can be managed with either melatonin or clonazepam. Two agents targeting alpha-synuclein (NPT200-11 and ambroxol) currently hold promise as disease-modifying therapies for DLB, but they are yet to be tested in clinical trials. An agent (E2027) that offers hope of neuroprotection by increasing central cyclic guanosine monophosphate (cGMP) levels is currently being examined in clinical trials in DLB patients.
\end{abstract}

\section{Key Points}

Cholinesterase inhibitors are important drugs for managing patients in all stages of dementia with Lewy bodies (DLB), since they improve global cognitive function and reduce visual hallucinations and other behavioral symptoms.

New data from a recent phase 2 clinical trial have demonstrated the effectiveness of zonisamide when used as an adjunct to levodopa in treating the parkinsonism associated with DLB.

Rapid eye movement sleep behavior disorder, a condition that may precede dementia and other core features of DLB, is often responsive to either melatonin or clonazepam.

Linda A. Hershey

linda-hershey@ouhsc.edu

1 Department of Neurology, University of Oklahoma Health Sciences Center, 920 Stanton L Young Blvd, Suite 2040, Oklahoma City, OK 73104-5036, USA

\section{Introduction}

Neurodegenerative diseases are the sixth leading cause of death in the United States, and dementia with Lewy bodies (DLB) is the third most prevalent (52 cases per 100,000 in the USA) behind Alzheimer's disease (AD) and Parkinson's disease (PD) [1]. When the incidence rate of DLB (3.5/100,000 person years) was compared to that of other common forms of parkinsonism, it was found to be second only to that of Parkinson's disease dementia (PDD) (2.5/100,000 person years) [2]. The median age of onset in DLB is significantly younger (76.3 years) than in PDD (81.4 years), and more men have DLB (4.8/100,000 person years) than women $(2.2 / 100,000$ person years) [2]. This review will discuss new imaging tools that can improve the clinical diagnostic accuracy of DLB, as well as describe new information about its genetics and pathophysiology. The main focus of this article is on the pharmacological management of the disease's various core clinical features: (1) dementia, (2) visual hallucinations, (3) parkinsonism, 
(4) fluctuations/agitation and (5) rapid eye movement (REM) sleep disorder. The sections about currently available therapies for DLB are followed by a discussion of investigational drugs that have been designed as diseasemodifying therapies.

\section{Diagnosis of Dementia with Lewy Bodies (DLB)}

\subsection{Clinical Criteria for DLB}

The presentation of DLB is usually one of both cortical and subcortical cognitive symptoms and signs, with worse visuospatial, executive and attentional dysfunction than is usually seen in AD. In the clinical series of Farina et al. [3], cognitive disturbances were reported at onset in $49 \%$ of their sample of 102 patients. Molano et al. [4] described memory loss in three of their eight mild cognitive impairment (MCI) patients of the DLB type (all eight of these MCI cases eventually had autopsy verification of their DLB). The other five cases had non-amnestic MCI (attention and executive problems were the most common early cognitive complaints). All of their patients had exhibited MCI complaints for 1-3 years before they met criteria for dementia, and they displayed the four associated symptoms of (1) REM sleep disorder, (2) parkinsonism, (3) visual hallucinations, and/or (4) fluctuations in attention. According to the fourth consensus report of the Dementia with Lewy Bodies Consortium, these four symptoms (besides dementia) are now considered to be the core clinical features of DLB [5]. Two or more of these four core features need to be present in order to make the diagnosis of probable DLB.

\subsection{Diagnosis of Dementia}

Dementia is defined as a decline in cognitive function of sufficient severity to impair one's usual daily activities [5]. The severity of cognitive impairment can be assessed in the clinic with screening tests such as the Mini-Mental State Examination (MMSE) or the Montreal Cognitive Assessment [6, 7]. Functional status can be evaluated with the Lawton-Brody Instrumental Activities of Daily Living (IADL) Scale or the Performance Assessment of Self-Care Skills [8]. Besides memory loss, the three cognitive domains most likely to be affected in early DLB are attention, visuospatial function and executive function [2, 4]. Cagnin et al. [9] compared DLB and AD patients and found that DLB patients showed poorer performance on the pentagon copying test and slower speeds on various visual processing tasks.

\subsection{Other DLB Symptoms}

There are other clinical features that distinguish DLB from AD. For example, DLB patients have been shown to be uniquely sensitive to neuroleptic drugs, since they are more likely to develop the neuroleptic malignant syndrome (NMS) in response to typical antipsychotic agents, such as haloperidol, or high-potency atypical agents, such as olanzapine [2, 10]. NMS is a hyperthermia syndrome that can be associated with worsening of signs of parkinsonism and rhabdomyolysis. Other symptoms of DLB include orthostatic hypotension, systematized delusions, excessive daytime sedation, anxiety, and depression [5]. DLB should be diagnosed when dementia precedes or appears concurrently with parkinsonism. If a patient with PD develops dementia a year or more after the onset of the parkinsonism, then this is called PDD.

\subsection{Imaging Diagnosis of DLB}

During the MCI stages of both MCI-DLB and amnestic MCI, hippocampal measurements on volumetric magnetic resonance imaging (MRI) scans show that the volumes are still within the normal range [11]. Once dementia develops in these two patient populations, differences appear in hippocampal volumes: atrophy becomes more evident in those with $\mathrm{AD}$, while volumes are more likely to remain in the normal range in DLB patients [11]. Hippocampal volumes may eventually become reduced over time in the DLB group, but at a much slower rate compared to that seen in AD. Complicating the diagnosis of DLB is the fact that most DLB cases also have concurrent AD changes at autopsy [12-14]. Sagittal views of volumetric MRI scans have demonstrated one feature that is similar in both DLB and AD patients: $25 \%$ atrophy in the basal forebrain [15]. Since many DLB patients exhibit poor visuospatial function, it is not surprising that perfusion single-photon emission computed tomography (SPECT) scans of DLB patients sometimes show reduced occipital perfusion, whereas occipital perfusion in AD patients is usually normal [16]. In both AD and DLB, there is usually reduced perfusion in both temporal and parietal lobes on perfusion SPECT images. Graff-Radford et al. [17] reported that DLB patients often have a unique sign on fluorodeoxyglucose positron-emission tomography (FDG-PET) called the "cingulate island sign," which means that there is higher glucose metabolism in the posterior cingulate, compared to that which is seen in AD patients. By contrast, in early AD, there is reduced metabolism in the posterior cingulate [18]. When perfusion SPECT and FDG-PET scans were compared for their sensitivity and specificity in distinguishing AD from DLB, FDG-PET scans were found to be superior [19]. Amyloid PET imaging, which has shown increased 
beta-amyloid deposition in 68\% of DLB patients, is not particularly useful in distinguishing AD from DLB patients, since both have significant amounts of amyloid deposits [20]. Dopamine transporter (DAT) scans are positive in both PD and DLB patients, but they can be helpful in distinguishing DLB from AD patients [5].

\section{Genetics of DLB}

Dementia and other core features of DLB have been shown to aggregate in families [3, 21, 22]. Tsuang et al. [21] found selected families where at least one member with dementia had autopsy evidence of neocortical and/or limbic Lewy body pathology. They found that these families differed as to whether signs of parkinsonism preceded or followed dementia. Nervi et al. [22] performed a familial cohort study in which DLB patients were recognized by core clinical features of cognitive fluctuations, visual hallucinations, and parkinsonism. Compared to siblings of probands having the clinical diagnosis of $\mathrm{AD}$, siblings of DLB patients had higher risks of having probable DLB (odds ratio 2.29; 95\% confidence interval (CI) 1.04-5.04). In a recent two-stage genome-wide association study using large numbers of DLB patients $(n=1743)$ and controls $(n=4454)$, the highest genetic risk for DLB was found at the apolipoprotein E (APOE) locus, whereas the second highest risk was found at the alpha-synuclein gene ( $S N C A$ ) locus [23]. A third genetic risk candidate for DLB is the glucocerebrosidase gene $(G B A)$, a common genetic risk factor for PD.

\section{Pathophysiology of DLB}

\subsection{Lewy Bodies}

Lewy bodies are abnormal spherical aggregates of protein that accumulate in neurons of the brains of DLB patients. Historically, a pathological diagnosis of DLB depended on the presence of brainstem, limbic, or cortical Lewy bodies. This allowed DLB cases to be classified as having one of three subtypes: brainstem, limbic (transitional) or neocortical. Later studies defined DLB according to whether Lewy bodies were identified in the locus coeruleus, substantia nigra, or nucleus basalis of Meynert, as well as in the neocortex [12]. A DLB/ PDD Working Group in 2007 agreed to use the term "Lewy body disorders" as an umbrella term to include PD, PDD and DLB, since efforts at drug discovery for one were likely to be shared by the others [24].

\subsection{Alpha-Synuclein}

Alpha-synuclein is the protein which is the major constituent of Lewy bodies. All of the Lewy body disorders (PD, PDD and DLB) involve abnormalities of alpha-synuclein metabolism [24]. Experimental studies have demonstrated that the pathological accumulation of alpha-synuclein into Lewy-body-like inclusions can lead to decreases in synaptic proteins, progressive impairment in neuronal excitability, and eventual neuronal death [25]. Cerebrospinal fluid (CSF) levels of alpha-synuclein have been shown to be lower in DLB patients than in those with other non-synucleinopathy dementias, suggesting that there is a "sink" of alpha-synuclein deposits in brain and in other systemic nervous system tissue. These observations lead Donadio et al. [26] to test for the presence of phosphorylated alpha-synuclein (p-syn) in skin biopsy material in 18 patients with DLB patients, 13 with AD, six with fronto-temporal lobe dementia, four with vascular dementia and 25 controls (the theory was that there would be enough autonomic nerve fibers in the skin biopsy material to demonstrate the presence of p-syn deposits). These authors found that p-syn was present in all of the skin samples of their DLB patients, but in none of the skin samples of those who had non-synucleinopathy dementia or who were controls. While these findings are of interest, the clinical diagnosis of DLB is still being made using the clinical criteria described by McKeith et al. [5].

\subsection{Tau}

Tau is a protein that stabilizes microtubules inside the neuron. In AD patients, tau protein aggregates and clumps into neurofibrillary tangles (Dr. Alzheimer showed these tangles with silver staining of the brain). Neurofibrillary tangles have been identified in DLB brains and have been shown to correlate with the severity of cortical and amygdala Lewy body changes [13]. Tau pathology was shown by these authors to be common in the olfactory lobes of AD and DLB patients and to correlate with the presence of the APOE-e4 genotype. The authors suggested a synergistic effect between tau and synuclein in the anterior olfactory nucleus in these patients. Mutations in the alpha-synuclein gene (SNCA) or overexpression of the gene have been associated with DLB [23, 27]. There is in vitro evidence showing that alpha-synuclein may serve as a seed to promote the aggregation of tau and that tau enhances the aggregation of alpha-synuclein [27]. Mice that overexpress the SNCA gene not only have brain inclusions that stain for alpha-synuclein, but they also have inclusions that stain for tau (some of these inclusions contain both proteins). This supports the idea that there appears to be synergy between the alpha-synuclein and tau changes in DLB brains. 


\subsection{Alzheimer's Disease Changes in DLB}

At autopsy, most DLB brains show signs of concomitant $\mathrm{AD}$, such as neurofibrillary tangles and amyloid plaques [12-14, 27, 28]. In a recent study of co-pathologies $(n=247$ AD cases and $n=164$ synucleinopathies), tau was found in almost all cases (92-100\%), amyloid-beta in a variable number (20-57\%), and alpha-synuclein was less common (4-16\%) [29]. These authors found that there was a high prevalence of co-pathology in neocortical Lewy body disease (70-81\%). They also found that the patient's APOE-e4 status was a risk factor for co-pathology of both AD and DLB findings. Merdes et al. [12] reported that the DLB patients who had fewer AD brain changes at autopsy had more visual hallucinations and parkinsonism during life, whereas those with more AD changes had more cognitive impairment and a longer survival. A different conclusion was reached by the study of Irwin et al. [28], which showed that as the levels of AD pathology increased, DLB disease duration became shorter. They observed that 49 (23\%) of the DLB patients had no AD changes, 56 (26\%) had mild AD changes, 45 (21\%) had moderate AD changes and 63 (30\%) had highlevel AD pathological changes. As levels of AD neuropathology increased, cerebral alpha-synuclein scores also became higher. There was a negative correlation between the cerebral neurofibrillary tangle score and the age of onset of motor and dementia symptoms; similarly, there was a negative correlation between the neurofibrillary tangle score and the duration of survival. These findings were consistent with the earlier ones of Tsuboi et al. [13], where there also appeared to be a synergistic relationship between tau and alpha-synuclein in DLB brains (the more severe the tauopathy, the earlier the onset of disease and the shorter the patient's survival).

\section{Treatments for Dementia in DLB}

\subsection{Cholinesterase Inhibitors}

Degeneration of cholinergic neurons in the basal forebrain appears in DLB brains, just as it does in AD brains [15]. These observations provided the rationale for several important clinical trials using cholinesterase inhibitors, where the goal was to improve cholinergic activity (and cognitive function) in patients with DLB (Table 1). Rivastigmine (oral dosages given up to $12 \mathrm{mg} /$ day) was the first to be tested in a large $(n=120)$ randomized, double-blind, placebo-controlled, international study [30]. Almost twice as many patients on rivastigmine (63\%) showed an improvement of $30 \%$ or more on a computerized cognitive exam, compared to those treated with placebo (where only $30 \%$ improved after 23 weeks). Patients on rivastigmine were also noted to be less anxious and to have fewer hallucinations. A similar pattern of change was observed with galantamine (oral dosages given up to $24 \mathrm{mg}$ / day) in a small $(n=50)$ open-label multi-center study of DLB patients, where there was significant improvement from baseline on the Clinician's Global Impression of Change (CGIC) scale (by +0.5 point $/$ total of 7 points; $p=0.01$ ) after 24 weeks [31]. The scores on the Neuropsychiatric Inventory (NPI-12) were reduced by a mean value of 8.24 points $(p=0.01)$; visual

Table 1 Medications for treating dementia with Lewy bodies

\begin{tabular}{|c|c|c|c|c|}
\hline Core feature & Drug class & Drug (generic) & Sample size $(n)$ & References \\
\hline \multirow[t]{5}{*}{ Dementia } & \multirow[t]{3}{*}{ Cholinesterase inhibitor } & Rivastigmine & 120 & {$[30]$} \\
\hline & & Galantamine & 50 & {$[31]$} \\
\hline & & Donepezil & 140 & {$[33]$} \\
\hline & \multirow[t]{2}{*}{ NMDA receptor antagonist } & Memantine & 72 & [36] \\
\hline & & & 199 & {$[37]$} \\
\hline \multirow[t]{5}{*}{ Hallucinations } & \multirow[t]{3}{*}{ Cholinesterase inhibitor } & Rivastigmine & 120 & {$[30]$} \\
\hline & & Galantamine & 50 & {$[31]$} \\
\hline & & Donepezil & 140 & {$[33]$} \\
\hline & Atypical antipsychotic (dopamine antagonist) & Quetiapine & 40 & [40] \\
\hline & Novel antipsychotic (5-HT2A inverse agonist) & Pimavanserin & - & {$[41]$} \\
\hline \multirow[t]{2}{*}{ Parkinsonism } & Dopamine precursor & Levodopa & - & {$[5,38,42]$} \\
\hline & Anticonvulsant & Zonisamide & 158 & {$[43]$} \\
\hline \multirow[t]{2}{*}{ Fluctuations and agitation } & Atypical antipsychotic (dopamine antagonist) & Quetiapine & - & {$[38,40]$} \\
\hline & Anticonvulsant & Valproic acid, divalproex & - & {$[50,52,53]$} \\
\hline \multirow[t]{3}{*}{ REM sleep behavior disorder } & Benzodiazepine & Clonazepam & - & {$[54,59]$} \\
\hline & Hormone & Melatonin & 110 & {$[60]$} \\
\hline & & & 14 & [61] \\
\hline
\end{tabular}

5-HT 5-hydroxytryptophan, NMDA N-methyl-D-aspartate, REM rapid eye movement 
hallucinations and nighttime behavior scores improved more than any of the other neuropsychiatric symptoms on that particular scale $(p=0.004)$. Parkinsonism (assessed with the Unified Parkinson's Disease Rating Scale [UPDRS]) did not become worse with cholinesterase treatment. By 2012, the conclusion of the Cochrane Database of Systematic Reviews [32] was that cholinesterase inhibitors were useful for PDD patients, but that the evidence for DLB was unclear (sample sizes in many of the trials had been small). This review was published before the large donepezil trial of Mori et al. [33] in July of that year. In that trial, oral donepezil was examined in a large group of DLB patients $(n=140)$ with a randomized, placebo-controlled study design. They found mean improvement on the MMSE to be 3.8 points (95\% CI $2.3-5.3 ; p<0.001$ ) after donepezil was given for 12 weeks at $5 \mathrm{mg} /$ day. Besides noting significant cognitive benefit, there were also significant changes in behavioral symptoms and caregiver burden. The side effects were dose dependent and the same as those seen with rivastigmine and galantamine (nausea, diarrhea, anorexia, and bradycardia). When Wang et al. [34] performed a systematic review and meta-analysis in 2015 concerning the safety and efficacy of cholinesterase inhibitors and memantine in DLB, they concluded that only the cholinesterase inhibitors showed significant benefit in cognitive function, while both classes of drugs showed improvement in clinical global impression of change.

\subsection{Memantine}

Glutamate is the main excitatory neurotransmitter in the brain. Animal models of parkinsonism and other neurodegenerative diseases have shown evidence for glutaminergic overactivity in cortical synapses [35]. Memantine is an $N$-methyl D-aspartate (NMDA) receptor antagonist that works in dementia patients by preventing the toxic effects of glutamate in the brain. Aarsland et al. [36] (Table 1) performed the first double-blind, placebo-controlled trial of memantine (oral dosages of $20 \mathrm{mg} /$ day) for both DLB and PDD patients $(n=72)$ and found that those treated with memantine had better CGIC scores at 24 weeks (mean difference was +0.7 point/total of 7 points; $p=0.03$ ). Emre et al. [37] designed a larger $(n=199)$ double-blind, placebocontrolled, 24-week trial with memantine (20 mg/day) and found that the drug produced significant improvement in CGIC scores in those with DLB (mean difference was 0.6; $p=0.02$ ), but not in those with PDD. They also found that the DLB patients treated with memantine experienced more improvement in behavior, compared to placebo patients $(-4.3$ vs 1.7 points on the NPI-12 scale; $p=0.041)$, while the PDD patients did not see significant change. There were no more adverse effects or discontinuations in the memantine group than in the placebo group. The lack of cognitive benefit seen in these two memantine trials is likely to be explained by the small sizes of their DLB sub-samples (these trials enrolled both DLB and PDD patients).

\section{Treatments for Hallucinations in DLB}

Vivid visual hallucinations are common in patients who have DLB. These phenomena can vary from "little people" who casually walk around the house, "ghosts" of dead parents who sit quietly at the bedside, to "bicycles" that hang off of trees in the back yard. In the early stages of the disease, the visual hallucinations may seem benign, but they may become more frightening to the patient as the disease progresses. In the autopsy series of Merdes et al. [12], $42 \%$ of their DLB patients reported visual hallucinations at some time during life. In the clinical series of Farina et al. [3], $44 \%$ reported hallucinations by the time of their first neurological consultation. In the epidemiological study of Savica et al. [2], visual hallucinations were more common during the course of DLB $(62.5 \%)$ than in those who carried the diagnosis of PDD (20\%).

\subsection{Cholinesterase Inhibitors}

In the mild to moderate stages of DLB, cholinesterase inhibitors like rivastigmine, galantamine and donepezil are usually effective in reducing the incidence and severity of delusions and hallucinations [30, 31, 33, 38] (Table 1). In the galantamine trial [31], for example, improvement in visual hallucinations and nighttime behaviors at 24 weeks $(p=0.004)$ exceeded that of the other ten items listed on the NPI-12. In a recent study performed in nursing homes in Sweden, the investigators found many residents who met clinical criteria for DLB were being treated with antipsychotic and antidepressant drugs rather than anti-dementia drugs [39]. The authors concluded that an increased recognition of DLB would lead to more effective prescribing of anti-dementia medications and a reduced need for psychoactive agents for the management of hallucinations and agitation in these patients.

\subsection{Atypical Antipsychotic Drugs (Dopamine Antagonists)}

When the psychotic symptoms of DLB patients become more threatening, the use of low doses of atypical antipsychotic drugs, such as quetiapine, should be considered [38]. Kurlan and others [40] (Table 1) performed a small clinical trial with dementia patients who were experiencing agitation or psychosis (23 of the 40 patients in this trial had DLB). They found that quetiapine (mean dosage $120 \mathrm{mg} /$ day) was welltolerated and did not worsen parkinsonism. Nevertheless, their study did not show demonstrable overall benefit of quetiapine in reducing agitation, hallucinations, or other signs of 
psychosis in dementia patients. See more discussion of this topic below under the section on treating agitation and cognitive fluctuations.

\subsection{Novel Antipsychotic Drug (5-HT2A Inverse Agonist)}

Pimavanserin is a selective 5-hydroxytryptophan (5-HT) 2A receptor inverse agonist that was recently approved by the Food and Drug Administration (FDA) for the treatment of psychosis in PD [41]. It has negligible effects on dopamine and histamine receptors, so it avoids the motor and sedative side effects that are commonly associated with other antipsychotic medications. It fills an important gap, since there are insufficient data to recommend the use of cholinesterase inhibitors to treat the psychosis seen in PD patients. Nevertheless, there are risks associated with pimavanserin, especially the prolongation of the QT interval (this drug should be avoided in patients with cardiac arrhythmias and in those taking other drugs prolonging the QT interval). Like other classes of antipsychotic drugs, there is an increased risk of death in elderly patients with dementia with pimavanserin. The National Institute on Aging is currently working with the pharmaceutical industry to sponsor a phase 3 clinical trial of pimavanserin for relapse of dementia-related psychosis (see http://www.clinicaltrials.org). Patients with DLB, PDD, or other dementias may qualify for the trial if they have had psychotic symptoms for at least 2 months and if they are on a stable dose of either memantine or a cholinesterase inhibitor.

\section{Treatments for Parkinsonism in DLB}

Parkinsonism is another common core clinical feature that distinguishes DLB from AD. The parkinsonism of DLB consists of bradykinesia and either rest tremor or rigidity. Dementia in DLB needs to occur before or within the first year of onset of the parkinsonism [5]. In their autopsy series, Merdes et al. [12] found that $55 \%$ of their DLB patients had parkinsonism at some point during their illness. In their clinical series of DLB patients, Farina et al. [3] demonstrated that parkinsonism was present in $22 \%$ of their patients at the time of neurological consultation and that most DLB parkinsonism was characterized as being symmetrical at onset (compared to the asymmetrical pattern of early PD).

\subsection{Levodopa}

The parkinsonism of DLB is often responsive to the dopamine precursor levodopa, but the dose needs to be kept low in order to avoid the aggravation of visual hallucinations or the appearance of agitation or excessive daytime sleepiness [38]. For example, Goldman et al. [42] showed that when levodopa was given to a group of 19 DLB patients, only four obtained significant motor benefit without exacerbation of their psychotic symptoms. In the DLB series of Farina et al. [3], the mean dosage of levodopa for the DLB parkinsonism was low (303 mg/day). Similarly, in the zonisamide trial of Murata et al. [43] for DLB parkinsonism, the mean dosage of levodopa was also low (319 mg/day).

\subsection{Zonisamide}

Zonisamide is an anticonvulsant drug that blocks sodium and T-type calcium channels; it also inhibits carbonic anhydrase and the release of glutamate. It has been approved in Japan for the treatment of PD. Murata et al. [43] (Table 1) designed a phase 2 clinical trial to test the safety and efficacy of zonisamide as an adjunct to levodopa in treating the parkinsonism associated with DLB. They enrolled 158 DLB patients into this trial from 60 institutions and showed that at a dosage of $50 \mathrm{mg} / \mathrm{day}$, there was significant motor benefit (as measured by the UPDRS part 3 motor subscale score) with zonisamide after 12 weeks. Several secondary outcome measures were used in their study, including cognitive, behavioral, and caregiver burden scales (zonisamide did not worsen cognitive function, behavioral symptoms, or caregiver burden). The most common side effects of zonisamide were decreased weight and decreased appetite, but these complaints were rare. A phase 3 trial of this drug in DLB patients is pending.

\subsection{Dopamine Agonists}

Dopamine agonist drugs such as pramipexole and ropinirole are often prescribed for young-onset PD patients, since these drugs are thought to be less likely than levodopa to result in the development of drug-induced motor fluctuations. Nevertheless, levodopa is preferred over dopamine agonist drugs in DLB because of the greater likelihood of the agonists causing hallucinations and somnolence [38].

\section{Treatments for Fluctuations and Agitation in DLB}

Fluctuations in alertness are a core clinical feature of DLB [5]. These fluctuations can be manifested as episodes of inattention ("zoning out"), excessive daytime sleepiness, confusional spells, stupor, incoherent speech, agitation, or delirium [47]. Fluctuating cognition can be seen in DLB, AD and vascular dementia, but it is more prevalent and more severe in those with DLB [44]. Farina et al. [3] found that cognitive fluctuations were present at the time of clinical presentation in $49 \%$ of their DLB patients. In the epidemiological study of Savica et al. [2], cognitive fluctuations were seen in $25 \%$ of DLB patients, compared to $9 \%$ of PDD patients. 


\subsection{Review of Medications and Medical History}

The DLB patient's medication list needs to be reviewed when new agitation, excessive daytime sedation, delirium, or other cognitive fluctuations appear, since opioids, anticholinergic drugs, benzodiazepines, and tricyclic antidepressant drugs have been shown to account for these symptoms [45]. New medical problems also need to be considered, since bladder infection, anemia, sleep apnea, upper respiratory illness, congestive heart failure, renal failure, or other systemic problems can lead to delirium or other cognitive fluctuations.

\subsection{Non-pharmacological Interventions}

Light is a powerful cue given by the environment to control the sleep-wake cycle. Merely exposing patients to window light is probably insufficient to improve their mood or to prevent cognitive fluctuations. Tailored lighting intervention, on the other hand, has been shown to improve mood, reduce agitation and increase total sleep time in $\mathrm{AD}$ patients and in those with other related dementias [46]. These authors used low-level bluishwhite lighting in the daytime in a nursing home setting. Further research is needed to demonstrate the optimal timing and intensity of light therapy that would have a long-term benefit in preventing cognitive fluctuations in DLB patients.

\subsection{Antipsychotic Drugs (Dopamine Antagonists)}

It is important to avoid using typical antipsychotic agents such as haloperidol and high-potency atypical agents such as olanzapine in DLB patients, since they are especially sensitive to these drugs, which can precipitate the NMS $[2,10]$. Some DLB patients, however, are likely to need low doses of an atypical antipsychotic drug such as quetiapine to manage their agitation and to prevent cognitive fluctuations [38]. In the series of Walker et al. [47], DLB patients had higher psychiatric symptom scores than AD patients when they were still in the mild stage of dementia (mean MMSE scores of 21/30). In this study, the authors found that $16 \%$ of mild DLB patients required neuroleptic medications to manage their agitation, compared to only $4 \%$ of mild $\mathrm{AD}$ patients. It is difficult, however, to verify the effectiveness of atypical antipsychotic drugs for DLB patients in clinical trials. When Schneider et al. [48] did a double-blind, placebo-controlled clinical trial to compare the benefits of three atypical antipsychotic drugs for the treatment of behavioral problems in AD, they found no significant differences between the effectiveness of risperidone, olanzapine, quetiapine, or placebo. A similar comparison trial has not been done with a large group of DLB patients, but Kurlan et al. [40] reported no behavioral benefit when quetiapine was compared to placebo (23 DLB patients were included in this study).

\subsection{Anticonvulsants}

Some DLB patients may present to an inpatient setting with stupor, agitation, or acute confusional spells [44]. Hershey et al. [49] described a mild DLB patient who developed delusions and agitation that could not be controlled with a low dosage of quetiapine ( $25 \mathrm{mg}$ three times a day); the dose of quetiapine could not be advanced further because of the patient's parkinsonism. The patient improved, however, when a low dosage of divalproex ( $250 \mathrm{mg}$ twice daily) was added as adjunctive therapy. Others have shown valproic acid to be effective as an adjunct to low doses of atypical antipsychotic drugs in managing the agitation associated with various dementing illnesses [50]. A systematic review performed by the Cochrane Database demonstrated that valproic acid (or divalproex) does not appear to be as useful for managing agitation in dementia patients when it is prescribed as monotherapy [51]. Nevertheless, more recent studies have demonstrated that valproic acid is effective as adjunctive therapy for agitation and delirium $[52,53]$. Both of these studies showed that by using valproic acid in acutely agitated patients, doses of opioids and other concomitant psychoactive medications could be reduced.

\section{Treatments for REM Sleep Behavior Disorder}

REM sleep behavior disorder (RBD) is now one of the core clinical features of DLB [5]. It is manifested by loss of normal muscle atonia during REM sleep, so that there is dream reenactment. In their original description of RBD, Schenck et al. [54] described four men who showed aggressive behaviors during sleep, which consisted of punches, kicks, and stereotyped hand motions. During non-REM sleep, there were no harmful behaviors, but three of the men had periodic myoclonus. Idiopathic RBD (iRBD) was shown by Postuma et al. [55] to be a long-term risk factor for the development of diseases such as PD and DLB. These authors observed that the estimated 5-year risk of neurodegenerative disease for iRBD was $17.7 \%$, while at 10 years, the risk was $40.6 \%$. The overall prevalence of iRBD in the general population is $0.4-0.5 \%$, while in DLB it is as high as $40 \%$ [56]. Ferman et al. [57] reported a series of DLB cases where dementia and iRBD initially appeared before the symptoms of either parkinsonism or hallucinations. They later demonstrated in a series of 234 autopsy-confirmed cases that inclusion of RBD as a core clinical feature improved the diagnostic accuracy of the disease [58].

\subsection{Clonazepam}

Clonazepam is a long-acting benzodiazepine. In their original RBD report, Schenck et al. [54] noted that two of their four patients experienced an immediate and long-lasting response 
of RBD symptoms to clonazepam. In both cases, withdrawal of the drug resulted in immediate return of symptoms. More recent $\mathrm{RBD}$ recommendations include modification of the sleep environment, since separate bedrooms have been shown to prevent injuries to the patient or to the sleep partner [59]. As many as 33-65\% of RBD patients have been reported to experience sleep related injuries. These authors also agreed that low dosages of clonazepam (0.5-1.0 mg every evening) decrease sleep-related injury in RBD patients, but they cautioned that the drug can cause adverse effects in those who have concomitant dementia, gait disorders, or untreated sleep apnea.

\subsection{Melatonin}

Melatonin is a hormone produced by the pineal gland which regulates sleep and wakefulness. McGrane et al. [60] (Table 1) reviewed three prospective $(n=68)$ and two retrospective $(n=42)$ studies using melatonin for patients who had polysomnographic evidence of RBD. The dosages of melatonin used were in the range of 3-9 mg/night. Each of these studies demonstrated that the drug effectively reduced the frequency and severity of RBD symptoms and signs. Boeve et al. [61] showed that DLB patients who had symptoms of RBD experienced improvement with melatonin at dosages ranging from 3 to $12 \mathrm{mg} /$ day. Their choice of melatonin was for those who had had a poor response to clonazepam, adverse effects from clonazepam, pre-existing dementia, narcolepsy, or the presence of sleep apnea. Five of their 14 cases experienced side effects with melatonin, including morning headaches, daytime sleepiness, and hallucinations. Eight patients noted persistent benefit with melatonin beyond 1 year of therapy. Larger trials will be needed.

\subsection{Nelotanserin}

Nelotanserin is a 5-HT2A inverse agonist that was initially developed for the treatment of chronic insomnia [62]. It is particularly effective at increasing total non-REM sleep time and delta-wave (deep) sleep, in addition to reducing multiple awakenings during sleep. Nevertheless, it failed to meet the needed endpoints in the phase 2 insomnia trial. Other investigators had already begun testing nelotanserin in DLB patients to see whether it might be useful for improving RBD, parkinsonism and other behavioral symptoms of DLB [63]. As of January 2018, the results of a small phase 2 DLB trial showed a positive trend, so that a larger confirmatory trial focusing on motor function and psychosis is now being planned.

\subsection{Intepirdine}

Another drug that functions as an antagonist at 5-HT2A receptors, intepirdine, is also a 5-HT6 receptor antagonist.
Drugs that work as antagonists at 5-HT6 receptors enhance the release of acetylcholine in the brain and improve alertness and memory in animal models [64]. A phase 2 clinical study of intepirdine in DLB, called the HEADWAY-DLB trial, started enrolling patients into a 6-month course of 35 or $70 \mathrm{mg} /$ day versus placebo in January of 2016. By January 2018, however, the sponsor reported negative results of the trial, and they discontinued development of the drug.

\section{Disease-Modifying Therapies for DLB}

There are currently several drugs in development that could potentially modify the course of DLB [63, 65-68]. There are also immunotherapies and combination therapies under investigation that could be used in later stages of the disease [69-71].

\subsection{Reducing Alpha-Synuclein}

Early interventions in DLB should focus on reducing toxic levels of alpha-synuclein. For example, there is an alphasynuclein misfolding inhibitor, NPT200-11, which has been shown in an animal model of PD to reduce two neuropathological signs of the disease: the accumulation of Lewy bodies and astrogliosis, which is a sign of neuroinflammation [66]. In addition to producing structural changes in the brain, NPT200-11 also normalizes striatal levels of the dopamine transporter in the brains of these mouse models of PD and improves their motor function. Another potential diseasemodifying therapy for PD and DLB is ambroxol, a drug that is currently available as treatment for respiratory diseases, since it breaks up phlegm and has anti-inflammatory properties [67]. It also increases the activity of the lysosomal enzyme glucocerebrosidase, which can lower central levels of the protein alpha-synuclein. The theory is that by reducing toxic levels of alpha-synuclein, both NPT200-11 and ambroxol will have the potential to slow progression of both PD and DLB. Ambroxol is currently being tested in a trial for PDD (see http://www.clinical trials.gov), but neither drug is being tested in DLB. A third drug with disease-modifying potential for DLB is E2027, a phosphodiesterase-1 inhibitor that is currently being tested in a 12 -week phase 2 clinical trial (see http://www.clinicaltrials.gov). The protective effects of phosphodiesterase-1 inhibitors as rescue agents from alphasynuclein toxicity appear to be mediated by increased levels of cyclic guanosine monophosphate (cGMP) [68]. Two primary outcome measures are being used in this trial: the Montreal Cognitive Assessment (MoCA) and the Clinician's Interview Based Impression of Change Plus Caregiver Input (CIBICPlus). Secondary outcome measures include NPI-12, MMSE and Cognitive Fluctuations Inventory (CFI) scores. The trial is scheduled to be completed on March 11, 2020. 


\subsection{Reducing Neuroinflammation}

The diagnosis of DLB is usually made when alpha-synuclein deposits are already established and neuroinflammation is widespread. Immunotherapy using antibodies to alpha-synuclein has been shown to be an effective way to reduce pathological and behavioral deficits in animal models of DLB [69]. There is difficulty, however, in the process of selecting the right antibodies to alpha-synuclein. The antibodies have to be able to pass the blood-brain barrier to affect the pathology in the brain. Another strategy to counteract the neuroinflammatory response in the synucleinopathies is active immunization with vaccination [70]. This approach is currently being evaluated in animal models of PD and multiple system atrophy. Other authors [71] have argued for combination therapy, where a drug would be used early to reduce the production of alpha-synuclein, then an immunotherapy intervention would be initiated later to prevent further neuronal loss.

\section{Conclusion}

DLB patients can now be clinically diagnosed using familiar tools that document cognitive and functional impairment. Besides dementia, DLB patients must have two of four core clinical features: visual hallucinations, parkinsonism, cognitive fluctuations, or RBD [5]. While there are no officially approved drugs for the treatment of DLB in the USA or the EU, donepezil is approved for use for DLB in Japan and the Philippines. There is good evidence to support the use of cholinesterase inhibitors in DLB for treating cognitive and behavioral deficits $[30,31,33]$. The benefit of memantine is limited to improving clinical global impression of change [36, 37], but the lack of cognitive benefit in these two trials may have been due to their small sample sizes. The parkinsonism of DLB is difficult to treat because of psychiatric side effects associated with levodopa use, but a recent phase 2 clinical trial has shown the benefit of using zonisamide as an adjunct to low doses of levodopa [43]. A phase 3 trial with this drug is still pending. Agitation in DLB often needs to be managed with low doses of atypical antipsychotic drugs [38, 47], but the dose may be limited due to motor side effects. Recent studies have shown that low doses of valproic acid (or divalproex) can be used as an adjunct to drugs like quetiapine in managing the agitation of DLB $[49,50,52,53]$. Pimavanserin is approved by the FDA for treating psychosis in PD, but it also needs to be tested in DLB patients who are experiencing hallucinations and delusions [41]. Clonazepam has been shown to be useful in preventing injury and to otherwise managing the disruptions of sleep in patients with RBD and DLB [54, $56,59,61]$. Several novel drugs are currently being tested in clinical trials as potential disease-modifying therapy agents for patients with DLB, including ambroxol [67] and phosphodiesterase-1 inhibitors [68].

\section{Compliance with Ethical Standards}

No violations of ethical standards occurred in the process of preparing this review.

Funding This article has received no external funding.

Conflict of interest Linda A. Hershey has received honoraria from MedLink Corporation for writing annual reviews on related topics ("Memory Loss" and "Sleep and Dementia"). She has received an honorarium from the American College of Physicians for reviewing a paper on a related topic. She sits on the American Academy of Neurology Workgroup that is currently preparing the Mild Cognitive Impairment Quality Measurement Set. She sits on the Oklahoma Longterm Care Stakeholder Committee for the Oklahoma Foundation for Medical Quality. Rhonda Coleman-Jackson declares that she has no conflicts of interest.

Open Access This article is distributed under the terms of the Creative Commons Attribution-NonCommercial 4.0 International License (http://creativecommons.org/licenses/by-nc/4.0/), which permits any noncommercial use, distribution, and reproduction in any medium, provided you give appropriate credit to the original author(s) and the source, provide a link to the Creative Commons license, and indicate if changes were made.

\section{References}

1. Mason AR, Ziemann A, Finkbeiner S. Targeting the low-hanging fruit of neurodegeneration. Neurology. 2014;83:1470-3.

2. Savica R, Grossardt BR, Bower JH, Boeve BF, Ahlskog JE, Rocca WA. Incidence of dementia with Lewy bodies and Parkinson disease dementia. JAMA Neurol. 2013;70(11):1396-402.

3. Farina E, Baglio F, Caffarra P, Magnani G, Scarpini E, Appollonio I, et al. Frequency and clinical features of Lewy body dementia in Italian memory clinics. Acta Biomed. 2009;80:57-64.

4. Molano J, Boeve B, Ferman T, Smith G, Parisi J, Dickson D, et al. Mild cognitive impairment associated with limbic and neocortical Lewy body disease: a clinicopathological study. Brain. 2010;133:540-56.

5. McKeith IG, Boeve BF, Dickson D, Halliday G, Taylor JP, Weintraub D, et al. Diagnosis and management of dementia with Lewy bodies. Neurology. 2017;89:88-100.

6. Tsoi KK, Chan JY, Hirai HW, Wong SY, Kwok TC. Cognitive tests to detect dementia: a systematic review and meta-analysis. JAMA Intern Med. 2015;175(9):1450-8.

7. Creavin ST, Wisniewski S, Noel-Storr AH, Trevelyan CM, Hampton T, Rayment D, et al. Mini-Mental State Examination (MMSE) for the detection of dementia in clinically unevaluated people aged 65 and over in community and primary care populations. Cochrane Database Syst Rev. 2016. https://doi.org/10.1002/14651858.cd011 145.pub2.

8. Ciro C, Anderson MP, Hershey LA, Prodan CI, Holm MB. Instrumental activities of daily living performance and role satisfaction in people with and without mild cognitive impairment: a pilot project. Am J Occup Ther. 2015;69:6903270020. https://doi.org/10.5014/ ajot.2015.015198.

9. Cagnin A, Busse C, Gardini S, Jelnic N, Guzzo C, Gnoato F, et al. Clinical and cognitive phenotype of mild cognitive impairment 
evolving to dementia with Lewy bodies. Dement Geriatr Cogn Disord Extra. 2015;5(3):442-9.

10. Teng PR, Yeh CH, Lin CY, Lai TJ. Olanzapine-induced neuroleptic malignant syndrome in a patient with probable dementia with Lewy bodies. J Neuropsychiatry Clin Neurosci. 2012;24(4):E1-2.

11. Kantarci K, Lesnick T, Ferman TJ, Przybelski SA, Boeve BF, Smith $\mathrm{GE}$, et al. Hippocampal volumes predict risk of dementia with Lewy bodies in mild cognitive impairment. Neurology. 2016;87:2317-23.

12. Merdes AR, Hansen LA, Jeste DV, Galasko D, Hofstetter CR, Ho GJ, et al. Influence of Alzheimer pathology on clinical diagnostic accuracy in dementia with Lewy bodies. Neurology. 2003;60:1586-90.

13. Tsuboi Y, Wszolek ZK, Graff-Radford NR, Cookson N, Dickson D. Tau pathology in the olfactory bulb correlates with Braak stage, Lewy body pathology and apolipoprotein epsilon4. Neuropathol Appl Neurobiol. 2003;29(5):503-10.

14. Schneider JA, Avanitakis Z, Bang W, Bennett DA. Mixed brain pathologies account for most dementia cases in community dwelling older persons. Neurology. 2007;69:2197-204.

15. Grothe MJ, Schuster C, Bauer F, Heinsen H, Prudlo J, Teipel SJ. Atrophy of the cholinergic basal forebrain in dementia with Lewy bodies and Alzheimer's disease dementia. J Neurol. 2014;261(10):1939-48.

16. Lobotesis K, Fenwick JD, Phipps A, Ryman A, Swann A, Ballard $\mathrm{C}$, et al. Occipital hypoperfusion on SPECT in dementia with Lewy bodies but not AD. Neurology. 2001;56(5):643-9.

17. Graff-Radford J, Murray ME, Lowe VJ, Boeve BF, Ferman TJ, Przybelski SA, et al. Dementia with Lewy bodies: basis of cingulate island sign. Neurology. 2014;83:801-9.

18. Minoshima S, Giordani B, Berent S, Frey KA, Foster NL, Kuhl DE. Metabolic reduction in the posterior cingulate cortex in very early Alzheimer's disease. Ann Neurol. 1997;42:85-94.

19. O'Brien JT, Firbank MJ, Davison C, Barnett N, Bamford C, Donaldson $\mathrm{C}$, et al. ${ }^{18} \mathrm{~F}$-FDG PET and perfusion SPECT in the diagnosis of Alzheimer and Lewy body dementias. J Nucl Med. 2014:55(12):1959-65.

20. Petrou M, Dwamena BA, Foerster BR, MacEachern MP, Bohnen NI, Muller ML, et al. Amyloid deposition in Parkinson's disease and cognitive impairment: a systematic review. Mov Disord. 2015;30(7):928-35.

21. Tsuang DW, DiGiacomo L, Bird TD. Familial occurrence of dementia with Lewy bodies. Am J Geriatr Psychiatry. 2004;12(2):179-88.

22. Nervi A, Reitz C, Tang MX, Santana V, Piriz A, Reyes D, et al. Familial aggregation of dementia with Lewy bodies. Arch Neurol. 2011;68(1):90-3.

23. Guerreiro R, Ross OA, Kun-Rodrigues C, Hernandez DG, Orme $\mathrm{T}$, Eicher JD, et al. Investigating the genetic architecture of dementia with Lewy bodies: a two-stage genome-wide association study. Lancet Neurol. 2018;17(1):64-74.

24. Lippa CF, Duda JE, Grossman M, Hurtig HI, Aarsland D, Boeve BF, et al. DLB and PDD boundary issues: diagnosis, treatment, molecular pathology, and biomarkers. Neurology. 2007;68(11):812-9.

25. Volpicelli-Daley LA, Luk KC, Patel TP, Tanik SA, Riddle DM, Steiber A, et al. Exogenous alpha-synuclein fibrils induce Lewy body pathology leading to synaptic dysfunction and neuron death. Neuron. 2011;72(1):57-71.

26. Donadio V, Insensi A, Rizzo G, Capellari S, Pantieri R, Maserati MS, et al. A new potential biomarker for dementia with Lewy bodies: skin nerve alpha-synuclein deposits. Neurology. 2017;89:318-26.

27. Galpern WR, Lang AE. Interface between tauopathies and synucleinopathies: a tale of two proteins. Ann Neurol. 2006;59:449-58.

28. Irwin DJ, Grossman M, Weintraub D, Hurtig HI, Duda JE, Xie SX, et al. Neuropathological and genetic correlates of survival and dementia onset in synucleinopathies: a retrospective analysis. Lancet Neurol. 2017;16(1):55-65.
29. Robinson JL, Lee EB, Xie SX, Rennert L, Suh E, Bredenberg $\mathrm{C}$, et al. Neurodegenerative disease concomitant proteinopathies are prevalent, age-related and APOE4-associated. Brain. 2018;141(7):2181-93.

30. McKeith I, DelSer T, Spano P, Emre M, Wesnes K, Anand R, et al. Efficacy of rivastigmine in dementia with Lewy bodies: a randomized, double-blind, placebo-controlled international study. Lancet. 2000;356(9247):2031-6.

31. Edwards K, Royall D, Hershey L, Lichter D, Hake A, Farlow M, et al. Efficacy and safety of galantamine in patients with dementia with Lewy bodies: a 24-week open-label study. Dement Geriatr Cogn Disord. 2007;23(6):401-5.

32. Rolinski M, Fox C, Maidment I, McShane R. Cholinesterase inhibitors for dementia with Lewy bodies, Parkinson's disease dementia and cognitive impairment in Parkinson's disease. Cochrane Database Syst Rev. 2012. https://doi.org/10.1002/14651858.cd006504. pub2.

33. Mori E, Ikeda M, Kosaka K, Donepezil-DLB Study Investigators. Donepezil for dementia with Lewy bodies: a randomized, placebocontrolled trial. Ann Neurol. 2012;72(1):41-52.

34. Wang HF, Yu JT, Tang SW, Jiang T, Tan CC, Meng XF, et al. Efficacy and safety of cholinesterase inhibitors and memantine in cognitive impairment in Parkinson's disease, Parkinson's disease dementia, and dementia with Lewy bodies: systematic review with meta-analysis and trial sequential analysis. J Neurol Neurosurg Psychiatry. 2015;86(2):135-43.

35. Warre R, Thiele S, Talwar S, Kamal M, Johnston TH, Wang S, et al. Altered function of glutaminergic cortico-striatal synapses causes output pathway abnormalities in a chronic model of parkinsonism. Neurobiol Dis. 2011;41(3):591-604.

36. Aarsland D, Ballard C, Walker Z, Bastrom F, Alves G, Kossakowski $\mathrm{K}$, et al. Memantine in patients with Parkinson's disease dementia or dementia with Lewy bodies: a double-blind, placebo-controlled, multi-centre trial. Lancet Neurol. 2009;8:613-8.

37. Emre M, Tsolaki M, Bonucelli U, Destee A, Tolosa E, Kutzelnigg A, et al. Memantine for patients with Parkinson's disease dementia or dementia with Lewy bodies: a randomized, double-blind, placebo-controlled trial. Lancet Neurol. 2010;9(10):969-77.

38. Fernandez HH, Wu CK, Ott BR. Pharmacotherapy of dementia with Lewy bodies. Expert Opin Pharmacother. 2003;4(11):2027-37.

39. Zahirovic I, Torisson G, Wattmo C, Londos E. Psychotropic and anti-dementia treatment in elderly persons with clinical signs of dementia with Lewy bodies: a cross-sectional study on 40 nursing homes in Sweden. BMC Geriatr. 2018;18(1):50.

40. Kurlan R, Cummings J, Raman R, Thal L, et al. Quetiapine for agitation or psychosis in patients with dementia and parkinsonism. Neurology. 2007;68(17):1356-63.

41. Hawkins T, Berman B. Pimavanserin: a novel therapeutic option for Parkinson disease psychosis. Neurol Clin Pract. 2017;7:157-62.

42. Goldman JG, Goetz CG, Brandabur M, Sanfilippo M, Stebbins GT. Effects of dopaminergic medications on psychosis and motor function in dementia with Lewy bodies. Mov Disord. 2008;23(15):2248-50.

43. Murata $M$, Odawara $T$, Hasegawa $K$, Iiyama $S$, Nakamura $M$, Tagawa M, et al. Adjunct zonisamide to levodopa for DLB parkinsonism: a randomized, double-blind phase 2 study. Neurology. 2018;90:e664-72.

44. Walker MP, Ayre GA, Cummings JL, Wesnes K, McKeith IG, O'Brien JT, et al. Quantifying fluctuation in dementia with Lewy bodies, Alzheimer's disease, and vascular dementia. Neurology. 2000;54(8):1616-25.

45. Clegg A, Young JB. Which medications to avoid in people at risk of delirium: a systematic review. Age Ageing. 2011;40:23-9.

46. Figueiro MG, Plitnick BA, Lok A, Jones GE, Higgins P, Hornick TR, et al. Tailored lighting intervention improves measures of sleep, depression, and agitation in persons with Alzheimer's disease and 
related dementia living in long-term care facilities. Clin Interv Aging. 2014;9:1527-37.

47. Walker Z, McKeith I, Rodda J, Qassem T, Tatsch K, Booij J, et al. Comparison of cognitive decline between dementia with Lewy bodies and Alzheimer's disease: a cohort study. BMJ Open. 2012;2:e000380.

48. Schneider LS, Tariot PN, Dagerman KS, Davis SM, Hsiao JK, Ismail MS, et al. Effectiveness of atypical antipsychotic drugs in patients with Alzheimer's disease. N Engl J Med. 2006;355(15):1525-38.

49. Hershey LA, Aggarwal R, Jindal P, Baranskaya I. Managing agitation in Alzheimer disease and related disorders. JSM Alzheimers Dis Relat Dement. 2014;1(2):1007.

50. Dolder CR, Nealy KL, McKinsey J. Valproic acid in dementia: does an optimal dose exist? J Pharm Pract. 2012;25:142-50.

51. Lonergan E, Luxenberg J. Valproate preparations for agitation in dementia. Cochrane Database Syst Rev. 2009. https://doi. org/10.1002/14651858.CD003945.pub3.

52. Sher Y, Miller Cramer AC, Ament A, Lolak S, Maldonado JR. Valproic acid for treatment of hyperactive or mixed delirium: rationale and literature review. Psychosomatics. 2015;56(6):615-25.

53. Gagnon DJ, Fontaine GV, Smith KE, Riker RR, Miller RR 3rd, Lerwick PA, et al. Valproate for agitation in critically ill patients: a retrospective study. J Crit Care. 2017;37:119-25.

54. Schenck CH, Bundlie SR, Ettinger MG, Mahowald MW. Chronic behavioral disorders of human REM sleep: a new category of parasomnia. Sleep. 1986;9(2):293-308.

55. Postuma RB, Gagnon JF, Vendette M, Fantini ML, MassicotteMarquez J, Montplaisir J. Quantifying the risk of neurodegenerative disease in idiopathic REM sleep behavior disorder. Neurology. 2009;72(15):1296-300.

56. Chan PC, Lee HH, Hong CT, Hu CJ, Wu D. REM sleep behavior disorder (RBD) in dementia with Lewy bodies (DLB). Behav Neurol. 2018;2018:9421098. https://doi.org/10.1155/2018/9421098.

57. Ferman TJ, Boeve BF, Smith GE, Silber MH, Lucas JA, GraffRadford NR, et al. Dementia with Lewy bodies may present as dementia and REM sleep behavior disorder without parkinsonism or hallucinations. J Int Neuropsychol Soc. 2002;8:907-14.

58. Ferman TJ, Boeve BF, Smith GE, Lin SC, Silber MH, Pedraza $\mathrm{O}$, et al. Inclusion of RBD improves the diagnostic accuracy of dementia with Lewy bodies. Neurology. 2011;77:875-82.

59. Aurora RN, Zak RS, Maganti RK, Auerbach SH, Casey KR, Chowdhuri $S$, et al. Best practice guide for the treatment of REM sleep behavior disorder (RBD). J Clin Sleep Med. 2010;6(1):85-95.
60. McGrane IR, Leung JG, St Louis EK, Boeve BF. Melatonin therapy for REM sleep behavior disorder: a critical review of evidence. Sleep Med. 2015;16(1):19-26.

61. Boeve BF, Silber MH, Ferman TJ. Melatonin for treatment of REM sleep behavior disorder in neurologic disorders: results in 14 patients. Sleep Med. 2003;4(4):281-4.

62. Al-Shamma HA, Anderson C, Chuang E, Luthringer R, Grottick AJ, Hauser E, et al. Nelotanserin, a novel selective human 5-hydroxytryptamine2A inverse agonist for the treatment of insomnia. J Pharmacol Exp Ther. 2010;332(1):281-90.

63. Velayudhan L, Ffytche D, Ballard C, Aarsland D. New therapeutic strategies for Lewy body dementias. Curr Neurol Neurosci Rep. 2017;17(9):68.

64. Colodny X, Vela JM, Ramirez MJ. 5-HT(6) receptor and cognition. Curr Opin Pharmacol. 2011;11(1):94-100.

65. Palermo G, Ceravolo R, Bonuccelli U. Advances in the pharmacotherapeutic management of dementia with Lewy bodies. Expert Opin Pharmacother. 2018;19(15):1643-53.

66. Price DL, Koike MA, Khan A, Wrasidlo W, Rockenstein E, Masliah E, et al. The small molecule alpha-synuclein misfolding inhibitor, NPT200-11, produces multiple benefits in an animal model of Parkinson's disease. Sci Rep. 2018;8(1):16165.

67. McNeill A, Magalhaes J, Shen C, Chau KY, Hughes D, Mehta A, et al. Ambroxol improves lysosomal biochemistry in glucocerebrocidase mutation-linked Parkinson disease cells. Brain. 2014;137(Pt5):1481-95.

68. Hollerhage M, Moebius C, Melms J, Chiu WH, Goebel JN, Chakroun $\mathrm{T}$, et al. Protective efficacy of phosphodiesterase-1 inhibition against alpha-synuclein toxicity revealed by compound screening in LUHMES cells. Sci Rep. 2017;7(1):11469. https://doi.org/10.1038/ s41598-017-11664-5.

69. Bergstrom AL, Kallunki P, Fog K. Development of passive immunotherapies for synucleinopathies. Mov Disord. 2016;31(2):203-13.

70. Schneeberger A, Tierney L, Mandler M. Active immunization therapies for Parkinson's disease and multiple system atrophy. Mov Disord. 2016;31(2):214-24.

71. Valera E, Masliah E. Combination therapies: the next logical step for the treatment of synucleinopathies? Mov Disord. 2016;31(2):225-34. 